\title{
Room Temperature Poling of Poly(Vinylidene Fluoride) with Deposited Metal Electrodes*
}

\author{
James M. Kenney and Steven C. Roth \\ Center for Materials Science, National Bureau of Standards, Washington, DC 20234 \\ May 21, 1979

\begin{abstract}
High values of pyroelectric and piezoelectric activity in fully-electroded films of poly(vinylidene fluoride) were obtained by "conventional" (non-corona) poling at room temperature with sufficiently high fields. The avoidance of breakdown while obtaining high activity requires an understanding of the time dependence of both breakdown and activity. Time-to-breakdown as a function of field, and room-temperature pyroelectric activity (7-14 days after poling at $23.5{ }^{\circ} \mathrm{C}$ ) as a function of poling time and field were obtained for $25-\mu \mathrm{m}$ biaxially stretched films with evaporated aluminum electrodes. The highest activities were obtained by poling at the highest fields and poling to breakdown. A pyroelectric activity of $36 \mu \mathrm{CK}^{-1} \mathrm{~m}^{-2}$ was measured two weeks after poling with a nominal field of $550 \mathrm{MVm}^{-1}$ for a nominal poling time of $10 \mathrm{~s}$ (reduced by multiple breakdown). The highest activity obtained with no apparent breakdown $\left(31 \mu \mathrm{CK}^{-1} \mathrm{~m}^{-2}\right)$ was measured a week after poling with a field of 400 $\mathrm{MVm}^{-1}$ for $10 \mathrm{~s}$. These values are comparable with the highest that have been reported for this material using any poling temperature or using corona poling.
\end{abstract}

Key words: Dielectric strength; electrets; piezoelectricity; polarization; poling procedures; polymers; poly(vinylidene fluoride); pyroelectricity; transducers.

\section{Introduction}

Many authors have sought, with varying degrees of success, to account for the pyroelectric and piezoelectric properties of poly(vinylidene fluoride) (PVDF). In the recent model by Broadhurst, et al. [1], ${ }^{1}$ these properties are directly proportional to remanent polarization. The achievement of a given degree of polarization, however, depends in a complex way, not yet understood, upon a number of variables associated with the poling process. Optimization of this process to achieve pyroelectric and piezoelectric activities that are both large and stable are therefore entirely empirical at this time.

One of the major obstacles in the development of poling techniques has been a strong tendency toward generalization based upon inadequate data. In particular, the following statements have in the past been commonly accepted:

1) An elevated temperature during poling is essential for high activity.

2) Poling is virtually complete after about 30 minutes.

3) Because form II ( $\alpha$-phase) crystallites are not polar they therefore cannot contribute to pyroelectric or piezoelectric activity.

* This paper was also a Final Report, to the Naval Ocean Systems Center, San Diego, California 92152, dated September 30, 1978 entitled "Fabrication of Piezoelectric Polymer Film."

${ }^{1}$ Figures in brackets indicate literature references at the end of this paper.
4) “. . . breakdown of electroded samples occurs at room temperature before fields high enough to pole the material can be reached [2]."

The "traditional" requirement of an elevated poling temperature has been largely demolished by the success of corona poling [2, 3]. Early studies [4-6] of the effect of poling time seemed to indicate a saturation of activity with time after 30 minutes or so, but Blevin [7] has shown how activity continues to increase (at $100 \mathrm{MVm}^{-1}$ ) over a long period, with faster poling rates at higher temperature. X-ray studies [3] of corona poled PVDF have indicated that form II $(\alpha$-phase) crystallites can be converted to a polar form, since the molecular chain does have a dipole moment. That fullyelectroded PVDF can be "conventionally" poled to high activity at room temperature was first reported, to our knowledge, by Sharp and Garn [8], who obtained 25 $\mu \mathrm{CK}^{-1} \mathrm{~m}^{-2}$ with a field of $300 \mathrm{MVm}^{-1}$ (in $6-\mu \mathrm{m}$ biaxially oriented PVDF with nichrome electrodes). A fuller exploration of the room temperature poling of fully-electroded samples is reported here.

\section{Procedures}

Initial attempts to increase the pyroelectric and piezoelectric activity of PVDF made apparent the importance of electrical breakdown as the limiting factor. Flashover to the walls of a brass sample cell used for elevated temperature 
poling, and large thermal inertia of the cell, were experimental difficulties which were eliminated with room temperature poling, for which no cell was needed. Large unelectroded margins of PVDF around the electrodes, to prevent edge flashovers, and the use of grease or oil over and around the electrodes, permitted very high voltages to be applied, resulting in higher pyroelectric and piezoelectric activity than was generally obtained by elevated temperature poling. Breakdown, however, appeared erratic, making difficult the achievement of high activity in undamaged samples. It became apparent that the breakdown at a given voltage depended on the time of application of that voltage, so a study was made of time-to-breakdown as a function of field.

Circular electrodes, generally aluminum $100 \mathrm{~nm}(1000 \AA)$ thick and $37 \mathrm{~mm}$ in diameter, were vacuum deposited by evaporation onto $25 \mu \mathrm{m}$ (nominal) films of biaxially stretched (blow molded, as supplied) "capacitor grade" PVDF from Kureha Chemical Co., Ltd. ${ }^{2}$ A large "tab" extension of each electrode allowed tinfoil leads to be attached with silverfilled rubber cement. Orientation of the tabs $180^{\circ}$ apart kept them out of the field, so that self-healing of the electrodes by evaporation of the aluminum during breakdown would not be inhibited by the attached leads.

A grounded guard ring, $136 \mathrm{~mm}$ inner diameter, was applied to the film around one electrode in order to intercept surface leakage. One electrode was connected to ground via a combination current and charge meter (designed for this work to have very low drift) which drove a dual-channel chart recorder.

To avoid instantaneous sample breakdown initiated by external flashover, it became necessary to immerse the samples in a light paraffin oil (Saybolt viscosity 125/135) when poling at high fields. The samples were later cleaned with methanol.

Following the breakdown study, other samples were poled with a wide range of fields for specific lengths of time using a microprocessor-type controller to program the poling voltage source. As one second was the minimum ramp time for this controller, the shortest poling time used was ten seconds at the nominal (constant) field. A different sample was used for each combination of poling time and field. Pyroelectric specimens of $28 \mathrm{~mm}$ diameter were punched from the poled samples and stored within folded tinfoil prior to their measurement between 7 and 14 days after poling. When breakdowns occurred, they almost invariably took place at the edges of the electrodes, except after many breakdowns (when damage progressed inward). The smaller diameter of the punched area, therefore, allowed the areas damaged by breakdown to be excluded from measurements. The pyroelectric activity of the punched specimens was determined by measuring the (reversible) current resulting from ramping the

${ }^{2}$ Identification of a commercial product is made only to facilitate experimental reproducibility and does not imply endorsement by NBS. sample temperature over a range of about two kelvin at close to room temperature, as described by Broadhurst, et al. [9]. A small irreversible component of sample current was compensated for by measuring currents for both increasing and decreasing temperatures and taking the difference between these at the same temperature. The assumption that the small temperature cycle did not alter the irreversible current was verified by comparing the pre-cycling and postcycling currents of several samples at the same steady temperature.

Previous to these measurements a number of samples, poled at room temperature at $100 \mathrm{MVm}^{-1}$ and $200 \mathrm{MVm}^{-1}$, had been measured for pyroelectric activity using the same current amplifier and also using a low-drift charge amplifier. These samples were also measured [9] for hydrostatic piezoelectric activity in the same sample cell, using the charge amplifier, with the cell temperature monitored to permit a correction for pyroelectric charge.

\section{Results}

As time did not permit the measurement of piezoelectric activity for most samples, it is useful to have a conversion factor between the pyroelectric coefficient, $p_{y}$, and hydrostatic piezoelectric coefficient, $d_{p}$, for this particular material, poled and measured at room temperature. As shown by figure 1 , the ratio of $d_{p}$ to $p_{y}$ is quite consistent to within the precision of measurement. The lower group of points represent samples poled at $100 \mathrm{MVm}^{-1}$, and the upper group

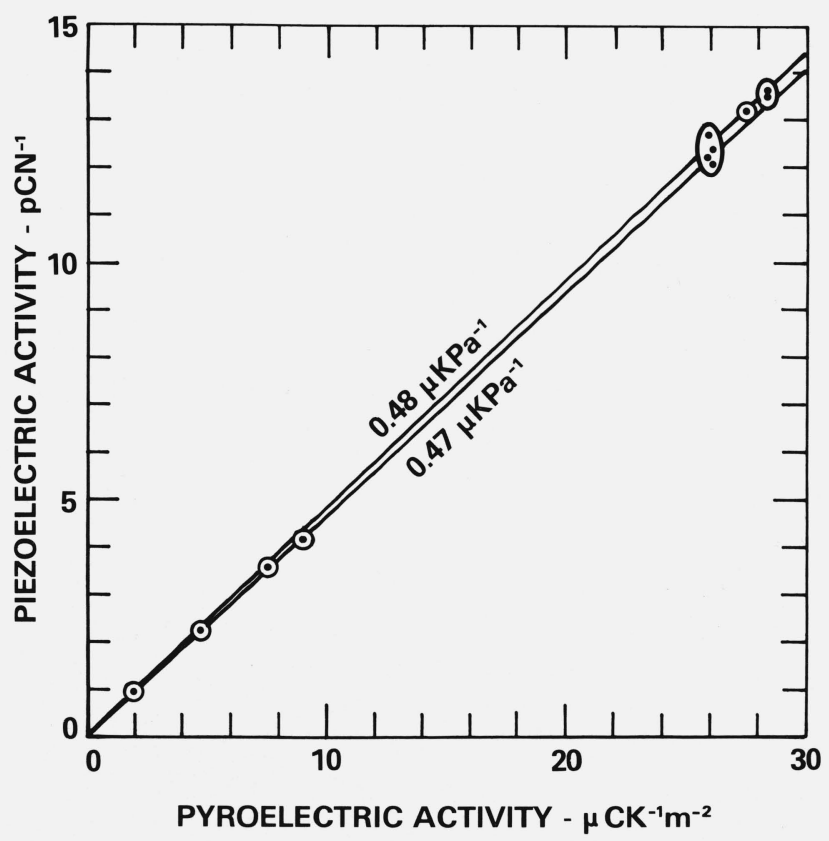

Figure 1. Determination of the ratio between hydrostatic piezoelectric activity and pyroelectric activity for samples poled and measured at room temperature. 
samples poled at $200 \mathrm{MVm}^{-1}$, with poling times ranging from $50 \mathrm{~s}$ to $3 \mathrm{~h}$. The average ratio of $d_{p}$ to $p_{y}$ is $0.478 \mu \mathrm{KPa}^{-1}$, with a sample standard deviation of 0.0157. Dropping the single most deviant datum (which also represented the lowest activity), the average ratio is $0.474 \mu \mathrm{KPa}^{-1}$, with a sample standard deviation of 0.0081 . These ratios are in good agreement with the predicted value [1] of $0.5 \mu \mathrm{KPa}^{-1}$. It must be noted, however, that subsequent poling was done at much higher fields. Virtually identical values of pyroelectric activity were obtained by measuring charge as by measuring current.

The breakdown data for aluminum-electroded samples is shown in figure 2; most of the data fit a straight line with a slope of $100 \mathrm{MVm}^{-1}$ per time decade. At the lowest field strengths there is an upward deviation, as would be expected, but the testing was not continued to fields low enough to closely approach a vertical asymptote (limit of time dependence). A common line seems to fit the data for both airpoled and oil-poled samples, although the latter appear to have a slightly longer time to breakdown at the same field. Above $420 \mathrm{MVm}^{-1}$ breakdown, even under oil, appeared to be instantaneous, probably occurring in most cases before the nominal voltage was reached. Subsequent poling of other samples for fixed times shorter than the breakdown times of figure 2 resulted in some rapid breakdowns at fields below

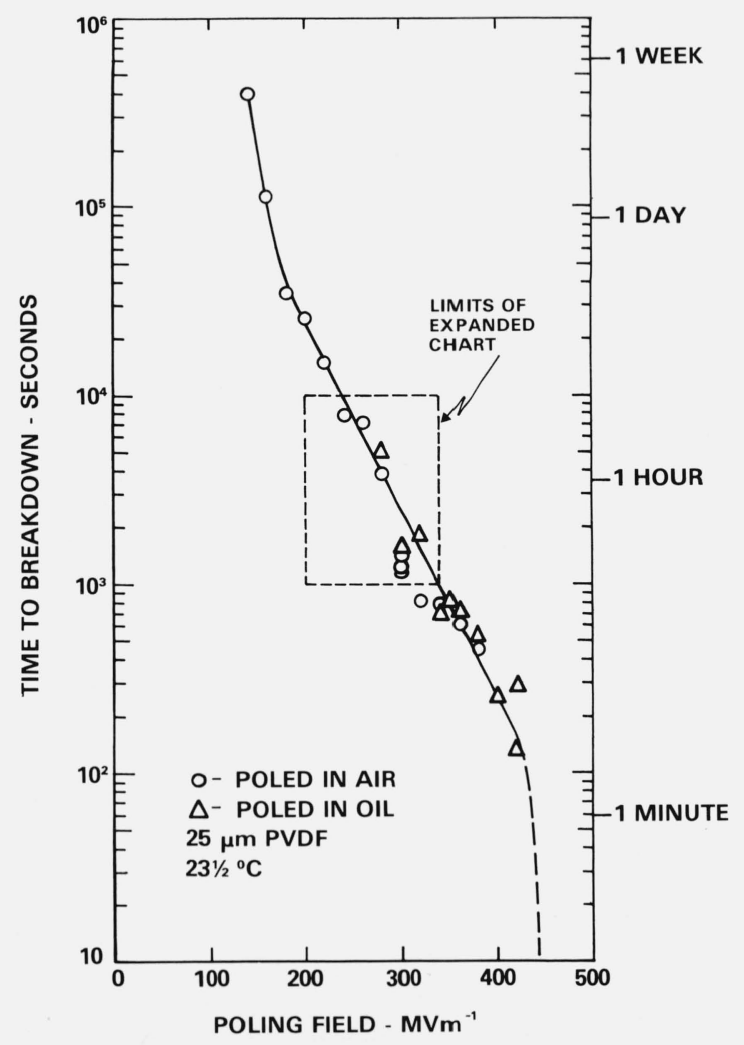

FIGURE 2. Time to breakdown as a function of field strength.
$420 \mathrm{MVm}^{-1}$, perhaps due to contamination of the oil from previous breakdowns or from exposure of the oil to the air. Improved techniques, such as oil degassing, or the use of a more suitable type of oil, may extend the straight portion of figure 2 to higher fields or at least provide better consistancy.

A number of samples with electrodes of metals other than aluminum were poled to breakdown in air at $300 \mathrm{MVm}^{-1}$. The results are shown in figure 3 , which is an enlargement of the area outlined near the center of figure 2 . Four aluminumelectroded samples were also poled to breakdown at this field, all of which, including one poled in oil, had unusually short breakdown times. There appears to be too much scatter to generalize about these electrode differences with regard to breakdown time. With regard to current during poling, however, the results are more conclusive, as shown by figure 4. As the field was first applied there was a large current which gradually dropped to an almost constant value, which is plotted in figure 4 , before gradually rising monotonically until breakdown occurred. The currents at breakdown and at fixed intervals after applying the field were also noted, but did not provide any more precise prediction of time to breakdown than does figure 2. Comparing work-functions [10] with the ordering of metals in figure 4 , the higher currents correspond to the larger work-functions, except for gold which is badly out of sequence (but which does not bond

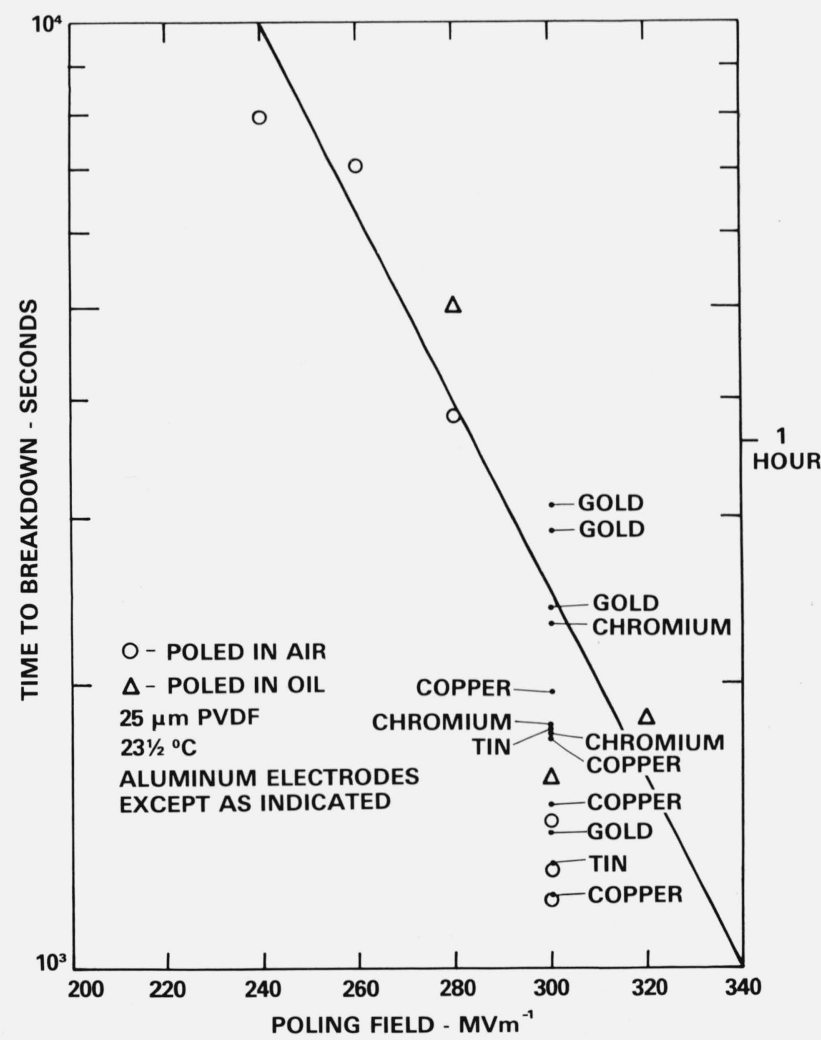

FiguRE 3. Enlarged portion of figure 2 with added data for samples electroded with various metals, as shown, and poled in air. 


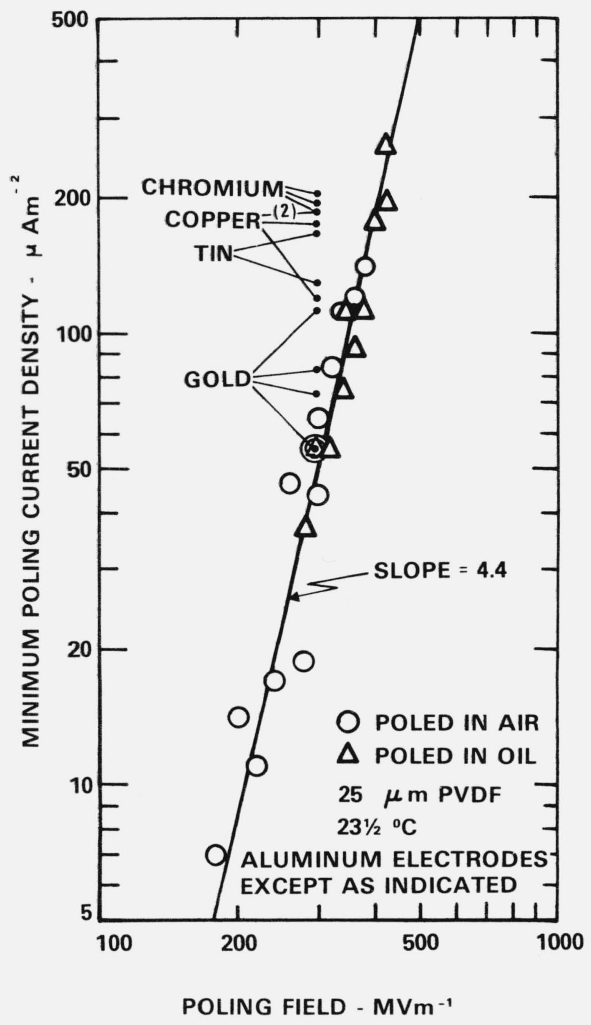

FIGURE 4. Minimum poling current density as a function of poling field.

well to PVDF). Superimposed on the slowly varying current were small and very narrow spikes, usually positive but frequently negative. The frequency (average spacing) of these spikes was reduced when poling in oil, so they are presumed to be associated with corona from exposed leads; this has not been studied, however.

The pyroelectric activity as a function of field strength of samples poled (generally) for times shorter than those of the curve in figure 2 are shown in figures 5 and 6 , the latter using decibel notation $\left(20 \log _{10} p_{y}\right)$ of arbitrary reference $(0$ $\left.\mathrm{dB}: 1 \mu \mathrm{CK}^{-1} \mathrm{~m}^{-2}\right)$ to provide a better indication of the significance of activity differences. The decibel increments should be the same for piezoelectric and pyroelectric activities. There is a discontinuity between data for air-poled and oil-poled samples, probably as a result of a smaller temperature rise in oil due to heating of the sample by the poling current. Considering separately the data above and below this discontinuity, there appears to be no abrupt saturation of activity with field in figure 5 , although, as shown by figure 6 , the relative increase in activity due to poling above 250 $\mathrm{MVm}^{-1}$ is only about $3 \mathrm{~dB}$, and may not be large enough in many applications to compensate for the problems of poling at higher fields. The highest-field sample $\left(550 \mathrm{MVm}^{-1}\right)$ experienced almost continuous breakdown, reducing the average poling field below the nominal value for most of the

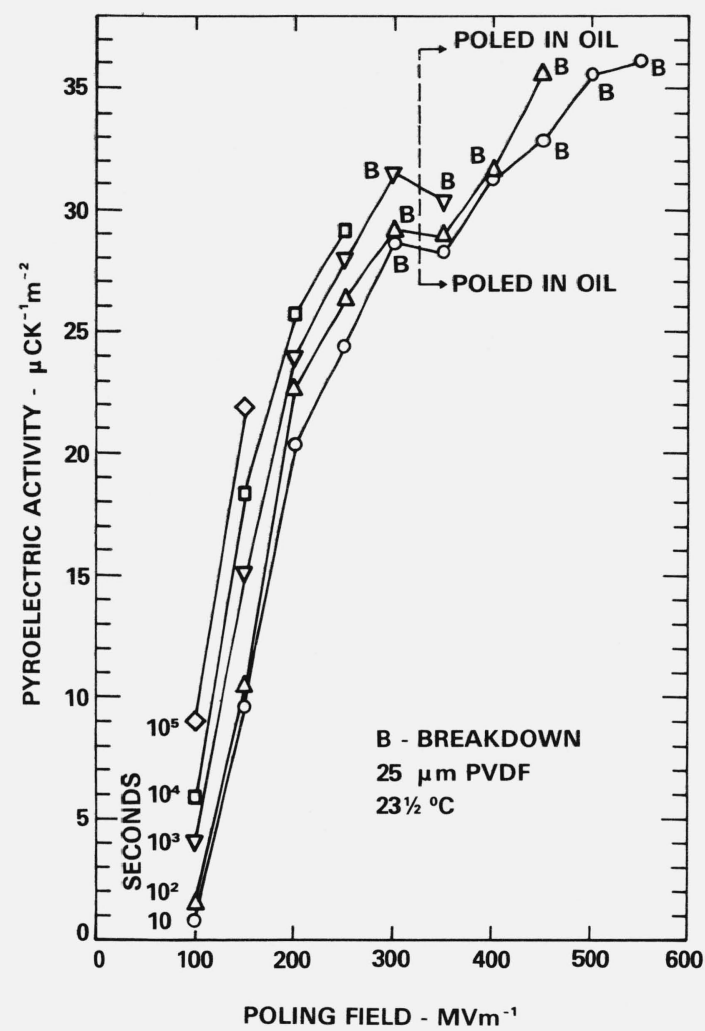

Figure 5. Pyroelectric activity as a function of poling field with poling time as a parameter.

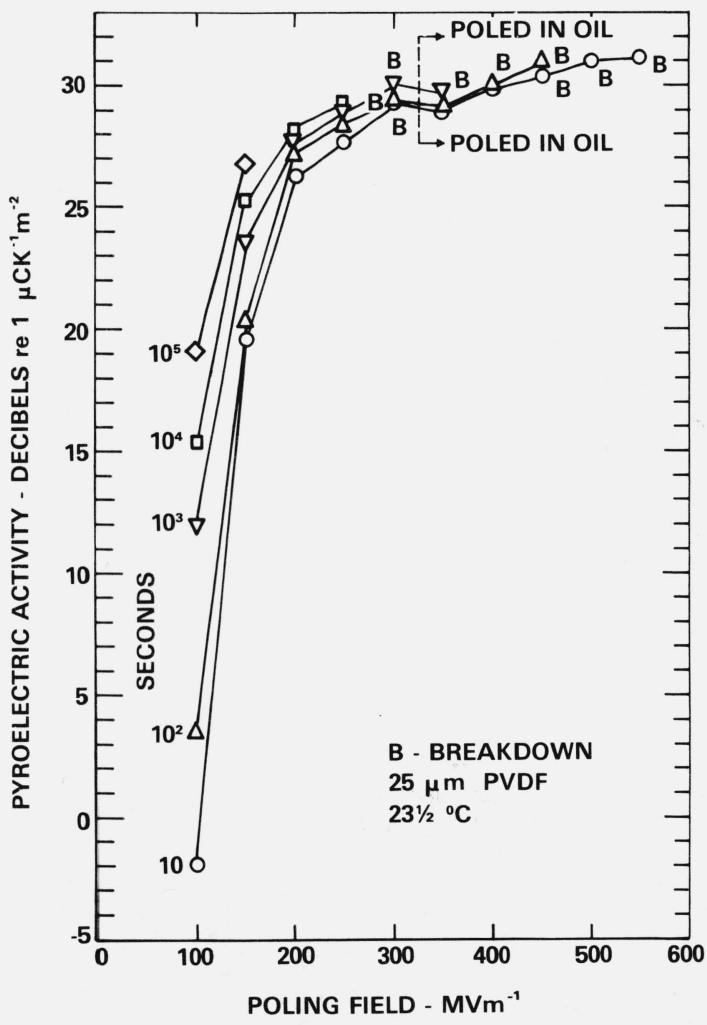

FIGURE 6. As in figure 5, but with the pyroelectric activity expressed in decibels. 


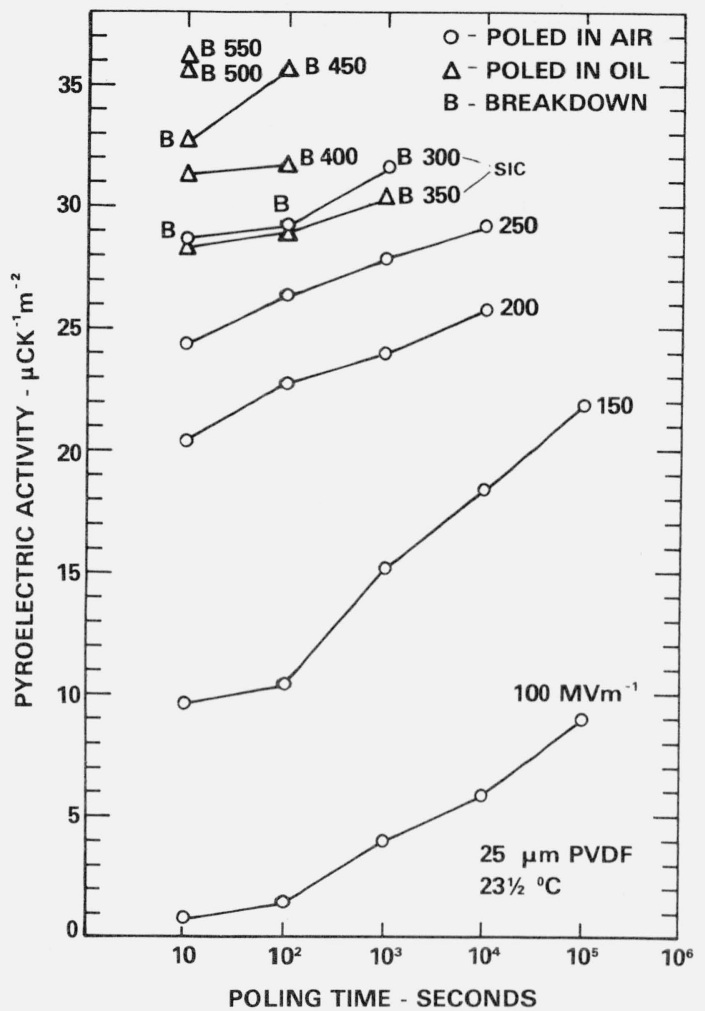

Figure 7. Pyroelectric activity as a function of poling time (on a log scale) with poling field as a parameter.

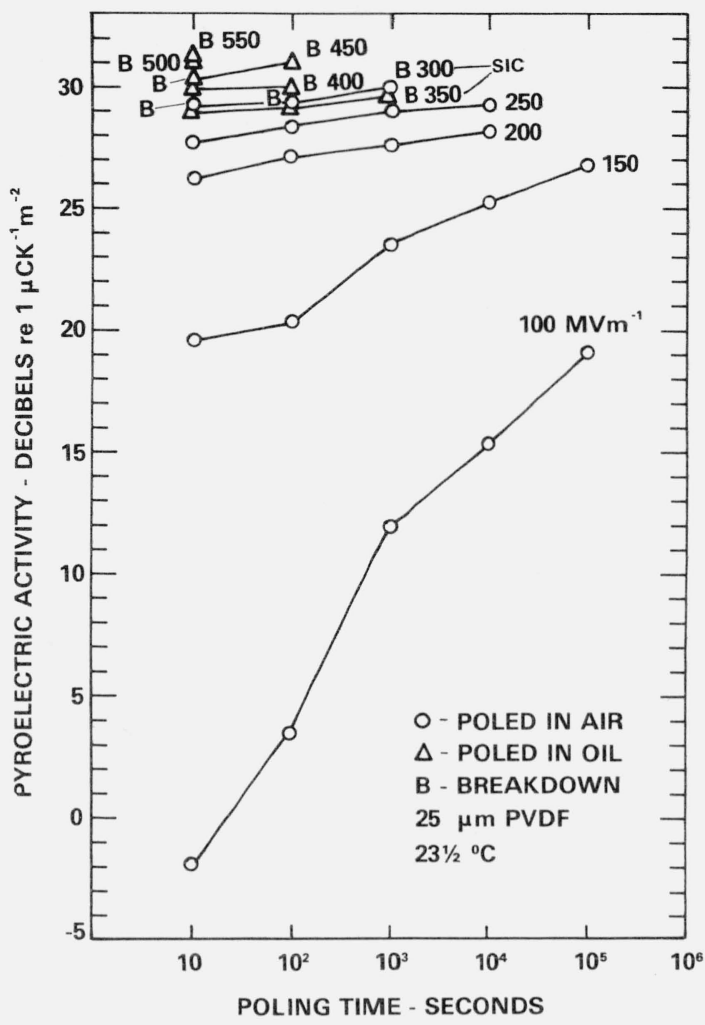

FIGURE 8. As in figure 7 , but with the pyroelectric activity expressed in decibels. poling time; this may account for the relatively small increase in activity relative to $500 \mathrm{MVm}^{-1}$. Breakdowns in other samples were generally isolated and probably caused little deviation from nominal conditions, especially for poling times of 100 seconds or more. The pyroelectric activity of these same samples as a function of poling time is shown in figures 7 and 8, the latter using decibel notation. As with field, there appears to be no abrupt saturation with time although at $200 \mathrm{MVm}^{-1}$ and above there is less than $1-\mathrm{dB}$ change in activity for each decade of poling time. At the highest fields, a scarcity of data and possible errors due to breakdowns preclude generalization.

By interpolation and extrapolation from the (unsmoothed) activity dependence plots, constant activity values were derived and combined with the breakdown curve (with extrapolations) of figure 2 to produce figures 9 and 10 . (Values derived from extrapolations are connected by broken lines). Of significance here are the intersections between the breakdown curve and the constant-activity curves. It is readily apparent that the highest activity is obtained at the highest fields, even though poling time may be severely limited by breakdown. The optimum poling conditions and the maximum obtainable activity depend upon the highest field strength that can be reached without an "instantaneous" breakdown and which, in turn, depends upon the precautions taken to prevent external flashovers that seem to initiate breakdowns. For the particular breakdown curve shown, with the "instantaneous" breakdown limit a vertical line, optimum poling time would be about $100 \mathrm{~s}$, since the small slope in the constant activity lines reduces the activity by a very slight amount for shorter poling times along the (vertical) limiting portion of the breakdown curve. For practical applications one may wish to avoid breakdown damage by poling outside the breakdown region by a reasonable safety margin. Pyroelectric activities approaching $30 \mu \mathrm{CK}^{-1} \mathrm{~m}^{-2}$ without breakdown appear to be possible. (In oil, $p_{y}>31$ $\mu \mathrm{CK}^{-1} \mathrm{~m}^{-2}$ was actually obtained with $400 \mathrm{MVm}^{-1}$ for $10 \mathrm{~s}$, and in air, $p_{y}>29 \mu \mathrm{CK}^{-1} \mathrm{~m}^{-2}$ was obtained with 250 $\mathrm{MVm}^{-1}$ for $10^{4} \mathrm{~s}$.) If breakdown damage can be tolerated, as when damaged areas can be trimmed off, even higher activities are possible. $\left(p_{y}>36 \mu \mathrm{CK}^{-1} \mathrm{~m}^{-2}\right.$ was obtained with $550 \mathrm{MVm}^{-1}$ for $10 \mathrm{~s}$, in oil, although damage was extensive, and $p_{y}>35 \mu \mathrm{CK}^{-1} \mathrm{~m}^{-2}$ was obtained with 450 $\mathrm{MVm}^{-1}$ for $100 \mathrm{~s}$, in oil, with only one small hole.)

Theory [1] predicts a linear dependence of pyroelectric (and piezoelectric) activity on remanent polarization. In figure 11, pyroelectric activity has been plotted against the total irreversible poling charge, i.e., the net charge that had passed through the external electrodes of the sample after the poling field had been reduced to zero and after the 


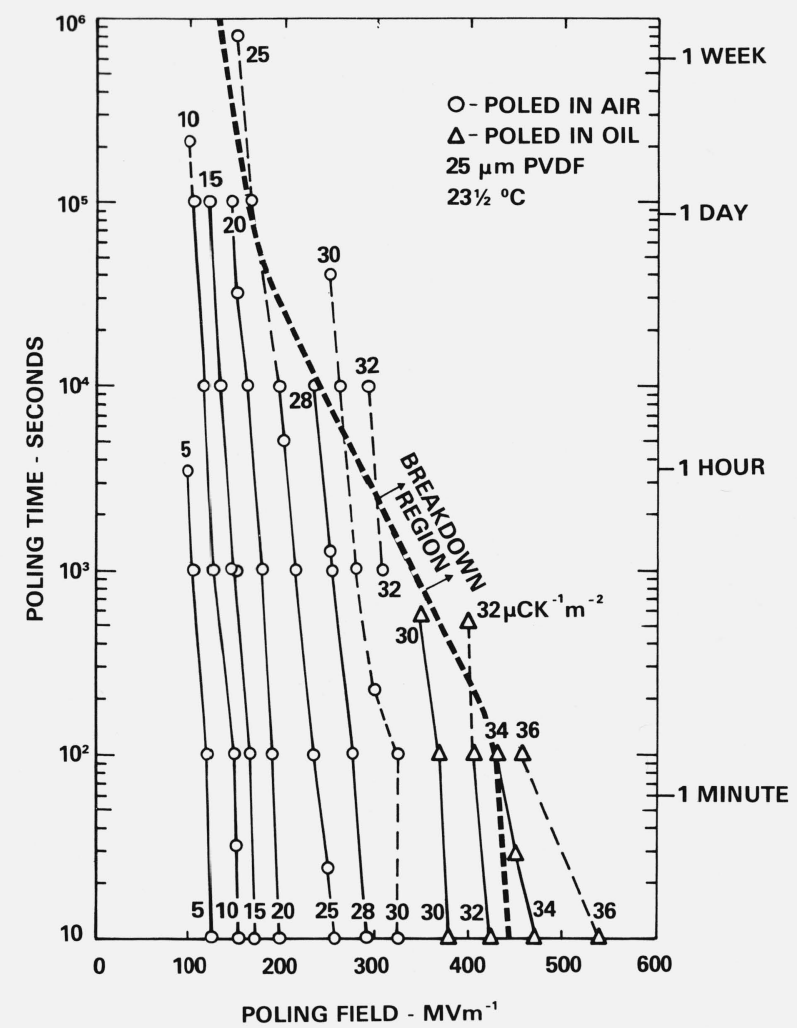

FIGURE 9. Constant pyroelectric activity curves, taken from figure 5 and 7 , and breakdown, taken from figure 2, as functions of poling field and poling time.

current transient had disappeared. This represents the remanent polarization plus the integral of the leakage current and instrumentation (error) currents. For many samples, this charge was not determined due to saturation of the first (current-to-voltage) stage of the current and charge meter by transients during either turn-on or turn-off of the poling supply, or to saturation of the integrator. When breakdowns occurred, a correction was made for charge lost (the usual case). Because of scaling problems and the need to avoid overload, the accuracy of the poling charge measurement was frequently poor, even neglecting error currents. It may be seen from figure 11 , however, that the activity of the samples poled at low-fields is reasonably proportional to charge. Notable exceptions include a $200 \mathrm{MVm}^{-1}$ sample where a poling time of $10^{4}$ seconds may have allowed a sizable charge accumulation from error currents. The other badly deviant samples were all poled at $300 \mathrm{MVm}^{-1}$ and above, and all experienced breakdown. Total current, however, was not visibly changed by breakdowns, following recovery, and a correction was made for any charge disturbance, as has been mentioned. Large error currents should result in a clear separation of points by poling time (and by field, for large leakage currents) but there is only slight evidence of such separation.

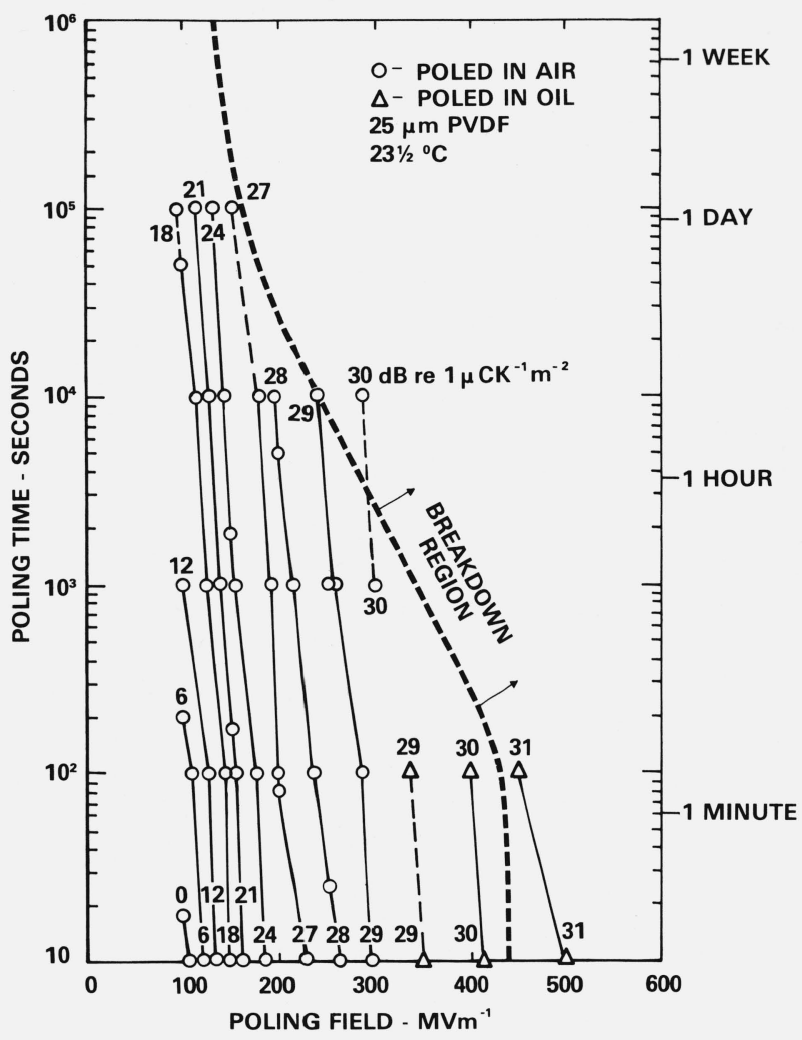

FIGURE 10. As in figure 9, but using pyroelectric activity expressed in decibels taken from figures 6 and 8.

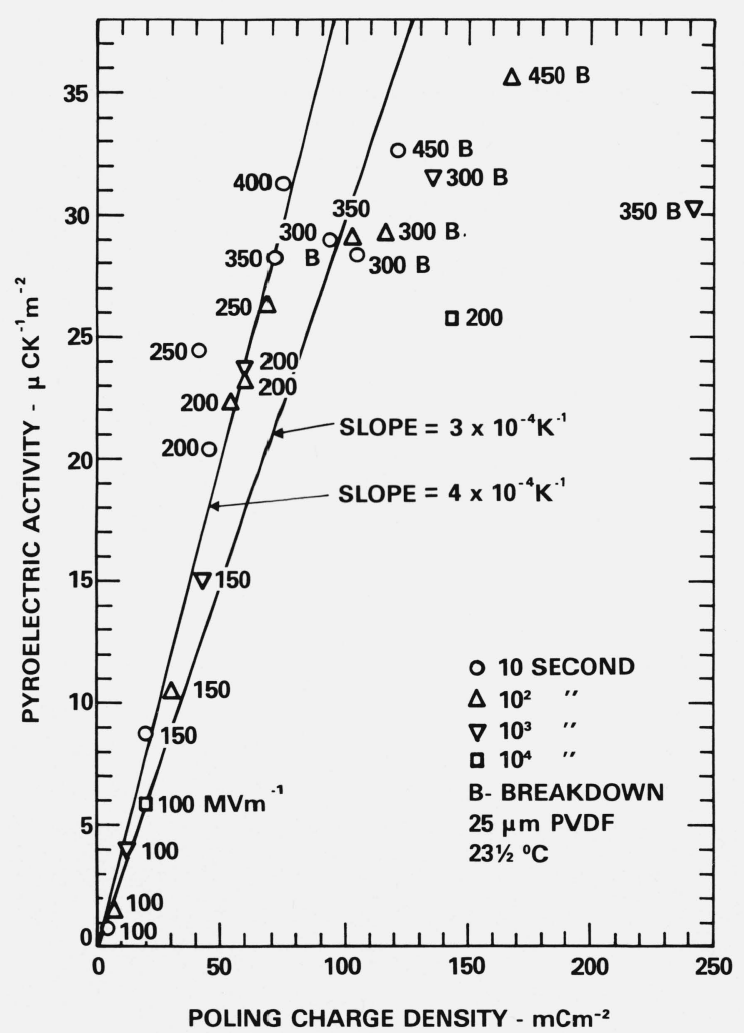

Figure 11. Pyroelectric activity as a function of poling charge density. 


\section{Conclusions}

High pyroelectric and piezoelectric activity can be obtained with biaxially stretched PVDF by poling fully-electroded samples at room temperature using a sufficiently high field. Time-to-breakdown and constant-activity plots can be used to optimize activity while avoiding or minimizing breakdown damage. Highest activity at other poling temperatures and for other materials can be obtained in similar fashion-by determining the time and field dependence of both activity and breakdown.

For the particular material investigated, poling at room temperature does not yield a hard saturation of activity with either field or time, although there is little practical benefit in using either the highest fields or the longest times. The lack of hard saturation may be due to the gradual alteration in crystal packing of TGTG' polymer chains from antipolar form II ( $\alpha$-phase) to polar alignment, as recently proposed [3], and perhaps at the highest field to a conversion to the all-trans polar form I ( $\beta$-phase).

There is no evidence that breakdown per se alters sample activity outside the damaged area, but it does limit both the field and the poling time.

Although room temperature is particularly convenient for poling, and may have commercial importance in the largescale production of poled material or in the fabrication of large "monolithic" transducer arrays, etc., higher activity may be attainable at some other temperature, although it has yet to be demonstrated that the improvement would be significant. A determination of the long-term stability of activity as a function of poling conditions would probably be more important than a small improvement in initial activity. Higher activity might be obtained by the use of much shorter poling times, which may allow higher fields to be applied. The large current needed to rapidly charge and discharge a sample of reasonable area, however, presents experimental difficulties. Finally, the dependence of activity on electrode material needs to be better understood.

Partial support of this work by the Naval Ocean Systems Center is gratefully acknowledged.

\section{References}

[1] Broadhurst, M. G., Davis, G. T., and McKinney, J. E., Piezoelectricity and pyroelectricity in polyvinylidene fluoride - a model. J. Appl. Phys. 49, 4992 (1978).

[2] Southgate, P. D., Room temperature poling and morphology changes in pyroelectric polyvinylidene fluoride. Appl. Phys. Lett. 28, 250 (1976).

[3] Davis, G. T., McKinney, J. E., Broadhurst, M. G., and Roth, S. C., Electric-field-induced phase charges in poly(vinylidine fluoride). J. Appl. Phys. 49, 4998 (1978).

[4] Murayama, N., Oikawa, T., Katto, T., and Nakamura, K., Persistent polarization in poly(vinylidene fluoride). II. Piezoelectricity of poly(vinylidene fluoride) thermoelectrets. J. Polymer Sci., 13, 1033 (1975).

[5] Shuford, R. J., Wilde, A. F., Ricca, J. J., and Thomas, G. R., Characterization and piezoelectric activity of stretched and poled poly(vinylidene fluoride). Part I: Effect of draw ratio and poling conditions. Polymer Eng. \& Sci. 16, 25 (1976).

[6] Day, G. W., Hamilton, C. A., Peterson, R. L., Phelan, R. J., Jr., and Mullen, L. O., Effects of poling conditions on responsivity and uniformity of polarization of $\mathrm{PVF}_{2}$ pyroelectric detectors. Appl. Phys. Letter. 24, 456 (1974).

[7] Blevin, W. R., Poling rates for films of polyvinylidene fluoride. Appl. Phys. Lett. 31, 8, (1977).

[8] Sharp, E. J., and Garn, L. E., Effects of aging on thermally stimulated currents in poly(vinylidene fluoride). Appl. Phys. Lett. 29, 480 (1976).

[9] Broadhurst, M. G., Malmberg, C. G., Mopsik, F. I., and Harris, W. P., Piezo- and pyroelectricity in polymer electrets. Electrets, Charge Storage and Transport in Dielectrics, M. M. Perlman, ed., Electrochem. Soc., Princeton, N. J. 492-504 (1973).

[10] Handbook of Chemistry and Physics, 47th ed., Chemical Rubber Co., Cleveland, Ohio, E67. 